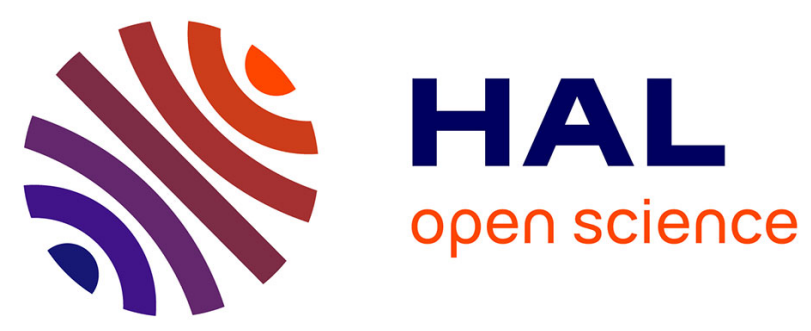

\title{
Fragmentation dynamics of ionized neon trimer inside helium nanodroplets: A theoretical study
}

David Bonhommeau, Alexandra Viel, Nadine Halberstadt

\section{To cite this version:}

David Bonhommeau, Alexandra Viel, Nadine Halberstadt. Fragmentation dynamics of ionized neon trimer inside helium nanodroplets: A theoretical study. Journal of Chemical Physics, 2004, 120 (24), pp.11359-11362. 10.1063/1.1763567 . hal-01118368

\section{HAL Id: hal-01118368 \\ https://hal.science/hal-01118368}

Submitted on 18 Feb 2015

HAL is a multi-disciplinary open access archive for the deposit and dissemination of scientific research documents, whether they are published or not. The documents may come from teaching and research institutions in France or abroad, or from public or private research centers.
L'archive ouverte pluridisciplinaire HAL, est destinée au dépôt et à la diffusion de documents scientifiques de niveau recherche, publiés ou non, émanant des établissements d'enseignement et de recherche français ou étrangers, des laboratoires publics ou privés. 


\title{
Fragmentation dynamics of ionized neon trimer inside helium nanodroplets: A theoretical study
}

\author{
David Bonhommeau, Alexandra Viel, and Nadine Halberstadt ${ }^{\mathrm{a}}$ ) \\ Laboratoire de Physique Quantique, IRSAMC, UMR 5626, CNRS et Université P. Sabatier, \\ 118 route de Narbonne, F-31062-Toulouse, France
}

(Received 8 April 2004; accepted 28 April 2004)

\begin{abstract}
We report a theoretical study of the fragmentation dynamics of $\mathrm{Ne}_{3}^{+}$inside helium nanodroplets, following vertical ionization of the neutral neon trimer. The motion of the neon atoms is treated classically, while transitions between the electronic states of the ionic cluster are treated quantum mechanically. A diatomics-in-molecules description of the potential energy surfaces is used, in a minimal basis set consisting of three effective $p$ orbitals on each neon atom for the missing electron. The helium environment is modeled by a friction force acting on the neon atoms when their speed exceeds the Landau velocity. A reasonable range of values for the corresponding friction coefficient is obtained by comparison with existing experimental measurements. (C) 2004 American Institute of Physics. [DOI: 10.1063/1.1763567]
\end{abstract}

\section{INTRODUCTION}

One of the most challenging problems in the recent field of molecular physics and chemistry inside helium nanodroplets is the question of how the helium environment affects molecular processes. In particular, since helium clusters have been shown to be superfluid, it is a fundamental question to know if dopant fragmentation can be hindered in this medium. It is an important practical question as well, since mass spectrometry is widely used for analyzing these systems: Electron impact ionization causes extensive fragmentation of the helium cluster and of the dopant. We focus here on the dopant fragmentation and how it is affected by the helium environment.

Isolated rare gas clusters are known for fragmenting upon ionization, ${ }^{1-3}$ due to the large difference between equilibrium distances in the neutral and ionized states. Therefore, they constitute ideal model systems to study the influence of the helium environment on a dissociative event. We have chosen to start our study with neon clusters since spin-orbit effects are expected to be small compared to heavier rare gases. There have been many experimental studies on isolated ionized neon clusters, in particular on the characterization of "magic numbers," 4 and on the ${ }^{22} \mathrm{Ne}$ isotope enrichment effect. ${ }^{5-7}$ Theoretical studies so far have been limited to the lowest adiabatic surface. Stampfli ${ }^{8}$ has studied the fragmentation of large ionized rare gas clusters by molecular dynamics and Satta et al. have modeled the neon trimer photoionization using time-dependent vibrational selfconsistent dynamics. ${ }^{9}$

Electron impact ionization and fragmentation of neon clusters inside helium clusters have been studied in detail by the group of Janda. ${ }^{10} \mathrm{Ne}_{3}$ and $\mathrm{Ne}_{4}$ clusters are found never to survive ionization. For both sizes, the most probable fragment is $\mathrm{Ne}_{2}^{+}$(bare or with some helium atoms attached). In the case of the tetramer, some $\mathrm{Ne}_{3}^{+}$fragments are also de-

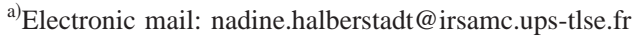

tected for the larger values of the mean helium droplet sizes.

In the present study we simulate the fragmentation of ionized neon clusters using the mixed quantum-classical dynamics (MDQT) method of Tully. ${ }^{11,12}$ The nuclei evolve classically on a single potential energy surface, and the existence of various potential energy surfaces is taken into account by allowing for hops between surfaces. The potential energy surfaces are based on the diatomics-in-molecules (DIM) description. The effect of the helium environment is modeled in an $a d$ hoc fashion by introducing a friction force on the neon atoms when their speed exceeds the critical Landau velocity. The justification is that helium clusters are superfluid at their temperature of $0.4 \mathrm{~K}$, but frictionless motion inside superfluid helium is valid only for speeds under the Landau velocity. ${ }^{13}$ Given the low temperature $(0.4 \mathrm{~K})$, the Langevin random force describing how energy is transferred from the helium bath to the neon cluster is neglected. In this paper we present the first results for $\mathrm{Ne}_{3}^{+}$inside helium nanodroplets, thereby demonstrating the applicability of the scheme to be used for larger clusters.

\section{THEORETICAL METHOD}

We use the molecular dynamics with quantum transitions (MDQT) method of Tully ${ }^{11,12,14,15}$ in which the electronic degrees of freedom are treated quantum mechanically and the nuclear ones classically. This method allows us to take into account all the potential energy surfaces and their couplings and makes it possible to study larger clusters. The classical motion of the nuclei is described in Cartesian coordinates on a single potential energy surface $V_{k}$ :

$$
\begin{aligned}
& \frac{\partial R_{\alpha l}}{\partial t}=\frac{p_{\alpha l}}{m}, \\
& \frac{\partial p_{\alpha l}}{\partial t}=-\frac{\partial V_{k}}{\partial R_{\alpha l}}-\gamma_{\alpha} \Theta\left(v_{\alpha}-v_{L}\right) v_{\alpha l},
\end{aligned}
$$


where $R_{\alpha l}$ and $p_{\alpha l}, \alpha=1, \ldots, n, l \in\{x, y, z\}$ respectively denote a Cartesian position and momentum component of neon atom $\alpha$ of mass $m=20.1797 \mathrm{u}$ (isotopic average). The second member in the r.h.s. of Eq. (1b) is a friction force, to be detailed below.

The time-dependent electronic wave function of the system is expanded on the adiabatic states $\Phi_{j}(r ; \mathbf{R})$,

$$
\Psi(r, \mathbf{R}, t)=\sum_{j=1}^{3 n} c_{j}(t) \Phi_{j}(r ; \mathbf{R}),
$$

where $\mathbf{R}$ and $r$ denote the set of classical variables $R_{i}$ and the electronic coordinates, respectively. The modulus $\xi_{j}(t)$ and the phase $q_{j}(t)$ of the complex-valued expansion coefficients $c_{j}(t)$ obey the following equations:

$$
\begin{aligned}
& \dot{\xi}_{j}=-\sum_{k \neq j} \xi_{k} \dot{\mathbf{R}} \cdot \mathbf{d}_{j k}(\mathbf{R}) \cos \left(q_{j}-q_{k}\right), \\
& \dot{q}_{j}=\frac{V_{j}}{\hbar}+\sum_{k \neq j} \frac{\xi_{k}}{\xi_{j}} \dot{\mathbf{R}} \cdot \mathbf{d}_{j k}(\mathbf{R}) \sin \left(q_{j}-q_{k}\right),
\end{aligned}
$$

where $\mathbf{d}_{j k}(\mathbf{R})=\left\langle\Phi_{j}(r ; \mathbf{R})\left|\nabla_{\mathbf{R}}\right| \Phi_{k}(r ; \mathbf{R})\right\rangle$ is the nonadiabatic coupling vector.

The multisurface aspect of the problem is taken into account by hops between surfaces for the classical coordinates. The probability to hop from state $k$ to state $j$ is given by ${ }^{11}$

$$
g_{k \rightarrow j}=-2 \Delta t \frac{\xi_{j}}{\xi_{k}} \dot{\mathbf{R}} \cdot \mathbf{d}_{j k}(\mathbf{R}) \cos \left(q_{j}-q_{k}\right),
$$

where $\Delta t$ is the current time step. The electronic wave function coefficients are not modified upon hopping. The atomic momenta are adjusted along the direction of the $\nabla\left(V_{k}-V_{j}\right)$ vector ${ }^{16}$ in order to ensure total energy and angular momentum conservation.

The electronic Hamiltonian $H_{\text {DIM }}$ of the neon cluster ion is derived from a DIM model ${ }^{17}$ with the addition of induced dipole-induced dipole interactions. It is expressed in the minimal basis set of $\mathrm{Ne}^{+}$effective $p$ orbitals ( 3 per atom) for the electronic hole. The potential energy curves required as inputs for the DIM Hamiltonian are taken from the literature: Ref. 18 for $\mathrm{Ne}_{2}$ and fit ${ }^{19}$ to recent ab initio calculations ${ }^{20}$ for the four $\mathrm{Ne}_{2}^{+}$potentials. The $3 n$ adiabatic state functions $\Phi_{j}(r ; \mathbf{R})$ and energies $V_{j}$ are obtained by diagonalizing $H_{\text {DIM }}$.

The helium environment is modeled by a friction force $\vec{f}_{\mathrm{He}}=-\gamma_{\alpha} \vec{v}_{\alpha}$ slowing down the nuclei if their speed $v_{\alpha}$ is superior to the Landau velocity $v_{L}=58 \mathrm{~m} / \mathrm{s}$, which corresponds to a kinetic energy of $2.8 \mathrm{~cm}^{-1}$ for a neon atom. ${ }^{13} \mathrm{In}$ Eq. (1b) $\Theta(\chi)$ is the Heaviside function, and the friction coefficient $\gamma_{\alpha}$ is taken to be proportional to the instantaneous charge $e_{\alpha}$ of the nuclei $\alpha: \gamma_{\alpha}=e_{\alpha} \gamma$. Different values for the friction coefficient are tested.

The initial conditions are selected in order to reproduce the experimental ones as closely as possible. Because of the low temperature $(\mathrm{T}=0.4 \mathrm{~K})$ of the helium nanodroplets, the cluster is assumed to be in its ground vibrational state. The classical equivalent condition is obtained as follows: One atom is pulled away from its minimum energy position along a randomly selected direction until the total energy of the
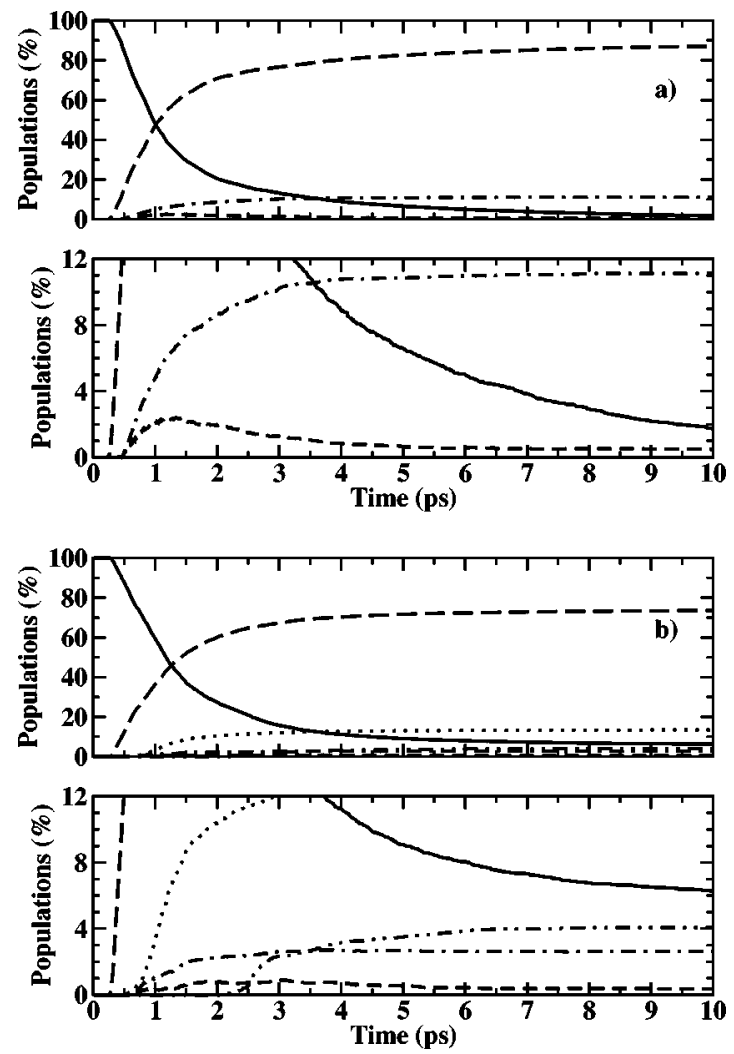

FIG. 1. Short time evolution (first $10 \mathrm{ps)} \mathrm{of} \mathrm{the} \mathrm{proportion} \mathrm{of} \mathrm{the} \mathrm{species}$ involved in the dissociation process of $\mathrm{Ne}_{3}$ cluster upon ionization for $\gamma$ $=0$ (a) and $\gamma=2.5$ a.u. (b): $\left(\mathrm{Ne}_{3}^{+}\right)^{*}$ (full line), $\mathrm{Ne}_{2}^{+}$(long-dashed line), $\mathrm{Ne}_{2}$ (dashed line), $\mathrm{Ne}^{+}$(dot-dashed line), $\mathrm{Ne}_{3}^{+}\left(\mathrm{A}^{\prime}\right)$ (double-dot-dashed) and $\mathrm{Ne}_{3}^{+}\left(\mathrm{A}^{\prime \prime}\right)$ (dotted). The $\mathrm{Ne}^{+}$curve includes population from both $(5 \mathrm{~b})$ and (5c) channels. Percentages have been averaged over 5000 trajectories.

cluster reaches its zero-point energy. The energy is allowed to thermalize during 2 ps by propagation on the potential energy surface of the neutral cluster. Ionization is then simulated by a vertical transition to a randomly selected ionic surface with a uniform distribution. This uniform probability of the initial electronic surface for the classical motion is mirrored in the electronic wave function by taking identical $c_{i}(t=0)$ coefficients [cf. Eq. (2)].

The time propagation of all the variables is carried out using the variable time step Hamming's predictor-corrector integrator initialized by a fourth-order Runge-Kutta method. An initial time step of $\Delta t_{(t=0)}=32$ a.u. $\approx 0.8$ fs is used. Fragmentation is assumed to occur when the minimum interfragment atom-atom distance is larger than $R_{c}=8 \AA$.

\section{RESULTS}

$\left(\mathrm{Ne}_{3}^{+}\right)^{*}$ clusters can a priori dissociate according to one of the three fragmentation channels:

$$
\begin{aligned}
& \left(\mathrm{Ne}_{3}^{+}\right)^{*} \rightarrow \mathrm{Ne}_{2}^{+}+\mathrm{Ne}, \\
& \left(\mathrm{Ne}_{3}^{+}\right)^{*} \rightarrow \mathrm{Ne}_{2}+\mathrm{Ne}^{+}, \\
& \left(\mathrm{Ne}_{3}^{+}\right)^{*} \rightarrow \mathrm{Ne}^{+}+\mathrm{Ne}+\mathrm{Ne} .
\end{aligned}
$$

Figure 1(a) shows the time evolution of the population of the species involved in the dissociation process of isolated $\left(\mathrm{Ne}_{3}^{+}\right)^{*}$, i.e., without helium environment $(\gamma=0)$. Channel 
TABLE I. Influence of the friction coefficient $\gamma$ (a.u.) on the proportion $(\%)$ of each $\left(\mathrm{Ne}_{3}^{+}\right)^{*}$ fragmentation channel. $\mathrm{Ne}_{3}^{+}$is determined to be stable when its total energy is below the $\mathrm{Ne}_{2}^{+}+\mathrm{Ne}$ dissociation limit. Five thousand trajectories are propagated during $100 \mathrm{ps}$ for each value of $\gamma$.

\begin{tabular}{|c|c|c|c|c|c|c|}
\hline$\gamma$ & 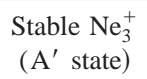 & $\begin{array}{r}\text { Stable } \mathrm{Ne}_{3}^{+} \\
\left(\mathrm{A}^{\prime \prime} \text { state }\right)\end{array}$ & $\mathrm{Ne}_{2}^{+}+\mathrm{Ne}$ & $\mathrm{Ne}_{2}+\mathrm{Ne}^{+}$ & $\mathrm{Ne}^{+}+\mathrm{Ne}+\mathrm{Ne}$ & $\begin{array}{l}\text { Time limit } \\
\text { reached }\end{array}$ \\
\hline 0. & 0 & 0 & $88.73 \pm 0.41$ & $0.38 \pm 0.12$ & $10.71 \pm 0.29$ & $0.18 \pm 0.06$ \\
\hline 0.025 & 0 & 0 & $89.58 \pm 0.46$ & $0.44 \pm 0.10$ & $9.84 \pm 0.54$ & $0.14 \pm 0.07$ \\
\hline 0.078 & 0 & 0 & $90.91 \pm 0.28$ & $0.50 \pm 0.10$ & $8.53 \pm 0.25$ & $0.06 \pm 0.03$ \\
\hline 0.25 & 0 & $0.72 \pm 0.10$ & $90.31 \pm 0.32$ & $0.38 \pm 0.06$ & $8.38 \pm 0.26$ & $0.20 \pm 0.05$ \\
\hline 0.75 & $0.18 \pm 0.04$ & $5.03 \pm 0.23$ & $88.61 \pm 0.56$ & $0.50 \pm 0.08$ & $4.93 \pm 0.43$ & $0.74 \pm 0.14$ \\
\hline 1.5 & $1.12 \pm 0.16$ & $10.23 \pm 0.48$ & $82.95 \pm 0.57$ & $0.36 \pm 0.08$ & $4.17 \pm 0.21$ & $1.16 \pm 0.15$ \\
\hline 2.5 & $4.07 \pm 0.22$ & $14.64 \pm 0.38$ & $77.45 \pm 0.49$ & $0.24 \pm 0.06$ & $2.30 \pm 0.24$ & $1.30 \pm 0.16$ \\
\hline
\end{tabular}

(5a) is found to be the main dissociative channel ( 88.73 $\pm 0.41 \%$ after a 100 ps propagation), which was also the case in the experimental studies conducted in Janda's group. ${ }^{10}$ Three-body dissociation $(5 \mathrm{c})$ is found to be the second largest channel $(10.71 \pm 0.29 \%)$. This process is sequential and mainly results from a previous two-body fragmentation in $\mathrm{Ne}_{2}$ or $\mathrm{Ne}_{2}^{+}$. The "hump" in the time evolution of the proportion of $\mathrm{Ne}_{2}$ around $1 \mathrm{ps}$ [Fig. 1(a)] is a signature of the transitory nature of this species. The very limited amount of $\mathrm{Ne}_{2}$ formed after 100 ps reflects the shallow well of $\mathrm{Ne}_{2}$. Since all the initial energies are above the $\mathrm{Ne}_{2}^{+}+\mathrm{Ne}$ dissociation limit, no stable $\mathrm{Ne}_{3}^{+}$is found. In their adiabatic study of $\mathrm{Ne}_{3}$ photoionization, Satta et al. ${ }^{9}$ report $21.5 \%$ of $\mathrm{Ne}_{3}^{+}$ after a 3-4 ps propagation. They have also found channel (5a) to be the most important one, but with a $\mathrm{Ne}_{2}^{+} / \mathrm{Ne}^{+}$ population ratio of about 1.9 instead of 8.3 in our case. The discrepancy is probably due to the approximations used in their model and to the fact that the dynamics is not over after 3-4 ps.

Addition of the friction force to model the helium environment modifies the evolution of the population of the different species involved, as shown in Fig. 1(b) for the largest $\gamma$ value tested (2.5 a.u.). The $\left(\mathrm{Ne}_{3}^{+}\right)^{*}, \mathrm{Ne}_{2}^{+}$, and $\mathrm{Ne}_{2}$ curves are similar to the isolated cluster case but with modified amplitudes. The initial decay of $\left(\mathrm{Ne}_{3}^{+}\right)^{*}$ is found to be slightly slower in the case with friction. This is due to the reduction of the particle speeds induced by the friction force, which makes the overall time scale for the dynamics longer. Another effect of the friction force is to stabilize the trimer by lowering the total energy below the $\mathrm{Ne}_{2}^{+}+\mathrm{Ne}$ dissociation limit. The two stable $\mathrm{Ne}_{3}^{+}$species of $\mathrm{A}^{\prime}$ and $\mathrm{A}^{\prime \prime}$ symmetry (of the $C_{s}$ group) are detected starting at about $0.5 \mathrm{ps}$ for the latter and $2.5 \mathrm{ps}$ for the former.

The effect of varying the friction coefficient $\gamma$ on the population of the different species obtained after a propagation of $100 \mathrm{ps}$ is summarized in Table I, together with the percentage of trajectories which have not led to stable fragments after 100 ps (last column of the table). Error bars have been estimated using ten separate sets of 500 trajectories each. The friction coefficient $\gamma$ has not been experimentally determined and is thus an unknown parameter in our model. We have varied its value between 0 and 2.5 a.u.. For comparison, the experimental value of the $\gamma$ coefficient is 2.5 $\times 10^{-3}$ a.u. for $\mathrm{Ne}^{+}$in gaseous helium at $4.35 \mathrm{~K},{ }^{21}$ and 0.078 a.u. for $\mathrm{Na}^{+}$in liquid helium at $1.42 \mathrm{~K}^{22}$
For all the tested $\gamma$ values, the dominant fragmentation channel is the formation of $\mathrm{Ne}_{2}^{+}$. For $\gamma$ below 0.25 a.u. no stable $\mathrm{Ne}_{3}^{+}$is detected after $100 \mathrm{ps}$, in contrast to the larger $\gamma$ values. Stable $\mathrm{Ne}_{3}^{+}$of $\mathrm{A}^{\prime}$ and $\mathrm{A}^{\prime \prime}$ symmetry reach a proportion of $4.07 \pm 0.22 \%$ and $14.64 \pm 0.38 \%$, respectively, for the largest value $\gamma=2.5$ a.u. They appear at different $\gamma$ values $\left(\gamma=0.25\right.$ a.u. for $\mathrm{A}^{\prime \prime}$ and $\gamma=0.75$ a.u. for $\left.\mathrm{A}^{\prime}\right)$. This stabilization of the parent ion cluster can be considered as a cage effect induced by the helium environment. In the Janda group's experiment, ${ }^{10}$ stable $\mathrm{Ne}_{3}^{+}$has not been observed, which places an upper bound of $5 \%$ on its proportion. ${ }^{23}$ The second main effect of increasing the friction is to decrease three-body fragmentation, and to increase $\mathrm{Ne}_{2}^{+}$formation (see Table I). This is a consequence of the reduction of the available kinetic energy which traps the system into potential wells. The number of "unfinished" trajectories increase with $\gamma$ (reaching $1.30 \%$ for the largest $\gamma$ value). They mostly correspond to a case of electronic metastability where the nuclei evolve on the highest excited $\mathrm{A}^{\prime \prime}$ surface close to its equilateral minimum. Finally, in most of the trajectories (from $98.8 \%$ for $\gamma=0.025$ a.u. to $99.9 \%$ for $\gamma=2.5$ a.u.) the neon atoms remain within the helium droplet for a droplet radius of $20 \AA$ ( $\approx 1000$ atoms). As a consequence, no droplet size effect is expected on the fragment populations, which is in agreement with the experimental findings for the trimer.

Since the $\mathrm{Ne}_{3}^{+}$parent ion has not been detected (proportion smaller than $5 \%),{ }^{10}$ we conclude that $\gamma$ should be less than 1.5 a.u. Because the observation of the $\mathrm{Ne}^{+}$fragment is difficult, ${ }^{10}$ the experimental population ratio of $\mathrm{Ne}^{+}$to $\mathrm{Ne}_{2}^{+}$ was not determined and cannot be used to determine a value for $\gamma$. Experiments on the fragmentation of isolated mass selected $\mathrm{Ne}_{3}$ upon ionization have not been done and comparison with the $\mathrm{He}_{N}$ embedded clusters results is thus not yet possible. However, if an effect of the helium environment is to be present, as was observed for $\mathrm{Ar}_{n}^{+}$clusters, ${ }^{24,25}$ then a lower limit for $\gamma$ of 0.025 a.u. can be inferred.

\section{CONCLUSIONS}

We have presented the first theoretical study of fragmentation of ionized rare gas clusters embedded in helium droplet. In this study the helium environment is modeled by a friction force acting on charged $\mathrm{Ne}$ atoms. A reasonable range of value for the unique parameter of the model, 
namely, the friction coefficient $\gamma$, has been set by comparison with available experimental data. In order to improve the determination of this parameter, calculations on larger cluster sizes for which experimental data are available are underway. The corresponding results are expected to constitute valuable information for the interpretation of experimental observations. The issue of the isotopic effects will also be addressed in future studies.

Extension of the model to include spin-orbit coupling is straightforward. It is necessary since we plan to study heavier rare gas clusters like $\mathrm{Ar}_{n}$ for which experimental data on fragmentation patterns for mass-selected isolated and embedded clusters are available. ${ }^{24,25}$

\section{ACKNOWLEDGMENTS}

The authors would like to express their thanks to Ken Janda and Marius Lewerenz for numerous and fruitful discussions. The Calmip computer center of Toulouse is gratefully acknowledged for a grant of computer time. The authors thank the University of Paul Sabatier, Toulouse, for financial support through an ATUPS grant (D.B.) and an APC grant (A.V. and N.H.).

${ }^{1}$ H. Haberland, Surf. Sci. 156, 305 (1985).

${ }^{2}$ U. Buck, J. Phys. Chem. 92, 1023 (1988).
${ }^{3}$ U. Buck, in The Chemical Physics of Atomic and Molecular Clusters, edited by G. Scoles (North Holland, Amsterdam, 1990), p. 543.

${ }^{4}$ T. D. Märk and P. Scheier, Chem. Phys. Lett. 137, 245 (1987).

${ }^{5}$ P. Scheier and T. D. Märk, J. Chem. Phys. 87, 5238 (1987).

${ }^{6}$ M. J. DeLuca, D. M. Cyr, W. A. Chupka, and M. A. Johnson, J. Chem. Phys. 92, 7349 (1990).

${ }^{7}$ M. Fieber, G. Bröker, and A. Ding, Z. Phys. D: At., Mol. Clusters 20, 21 (1991).

${ }^{8}$ P. Stampfli, Z. Phys. D: At., Mol. Clusters 40, 345 (1997).

${ }^{9}$ M. Satta and F. A. Gianturco, Mol. Phys. 100, 3699 (2002).

${ }^{10}$ T. Ruchti, K. Förde, B. E. Callicoatt, H. Ludwigs, and K. C. Janda, J. Chem. Phys. 109, 10679 (1998).

${ }^{11}$ J. C. Tully, J. Chem. Phys. 93, 1061 (1990).

${ }^{12}$ S. Hammes-Schiffer and J. C. Tully, J. Chem. Phys. 101, 4657 (1994).

${ }^{13}$ D. R. Allum, P. V. E. McClintock, A. Phillips, and R. M. Bowley, Philos. Trans. R. Soc. London 284, 23 (1977).

${ }^{14}$ W. Müller and G. Stock, J. Chem. Phys. 107, 6230 (1997).

${ }^{15}$ M. D. Hack and D. G. Truhlar, J. Phys. Chem. A 104, 7917 (2000).

${ }^{16}$ N. C. Blais and D. G. Truhlar, J. Chem. Phys. 79, 1334 (1983).

${ }^{17}$ F. Y. Naumkin and D. J. Wales, Mol. Phys. 93, 633 (1998).

${ }^{18}$ R. A. Aziz and M. J. Slaman, Chem. Phys. 130, 187 (1989).

${ }^{19}$ The parameters of the fit are available upon request to the authors.

${ }^{20}$ T. H. Ha, P. Rupper, A. Wüest, and F. Merkt, Mol. Phys. 101, 827 (2003).

${ }^{21}$ N. Saito, T. M. Kojima, N. Kobayashi, and Y. Kaneko, J. Chem. Phys. 100, 5726 (1994).

${ }^{22}$ H. Günther, M. Foerste, G. zu Putlitz, and T. Schumacher, Low Temp. Phys. 22, 143 (1996).

${ }^{23} \mathrm{~K}$. C. Janda (private communication).

${ }^{24}$ U. Buck and H. Meyer, J. Chem. Phys. 84, 4854 (1986).

${ }^{25}$ B. E. Callicoatt, K. Förde, T. Ruchti, L. Jung, K. C. Janda, and N. Halberstadt, J. Chem. Phys. 108, 9371 (1998). 